\title{
Multimodal Characterization of the Oleophilic Hydrophobic Magnetic (OHM) Sponge: A Nanocomposite Material for Oil Spill Remediation
}

\author{
Stephanie Ribet, Vikas Nandwana, Roberto dos Reis, Tirzah Abbott, Eric Roth and Vinayak Dravid
}

Northwestern University, Evanston, Illinois, United States

The Oleophilic Hydrophobic Magnetic (OHM) synthetic sponge is a novel nanocomposite system developed in the Dravid lab that offers the ability to repeatedly adsorb 20-35 times its weight in oil. [1,2] The OHM sponge is synthesized by coating a polyurethane sponge (soft) with a nanocomposite, made of mixed metal oxide nanoparticles, such as $\mathrm{Fe}_{3} \mathrm{O}_{4}$, and graphite (hard). We have demonstrated exceptional macroscale oleophilicity and hydrophobicity both in oil remediation tests and in isolated, controlled contact angle tests. In both experiments, we have observed robust hydrophobicity over the course of multiple cycles.

The hydrophobic nature of a material is determined by both its surface chemistry and its physical structure. In order to unravel the factors underpinning the OHM sponge's unusual hydrophobic nature, we characterized the unique interfaces of this composite material in the context of solid-fluid interactions using a correlative approach. Here we apply a combination of SEM, TEM and Raman spectroscopy to probe the structure, morphology and chemistry of the sponge at multiple length scales. This correlative and multimodal approach is fundamental to fully depict the structure-property relationship of the OHM sponge and overcome the intrinsic challenges of studying hard-soft interfaces. [3-5]

Figure 1 shows an SEM and bulk (inset) image of the sponge before (a) and after (b) the coating is applied. These images show that the OHM sponge has pore diameter on the order of $100 \mu \mathrm{m}$, which makes assessing the OHM sponge at the micro and nano scale an essential component in disentangling the chemical and physical properties that contribute to its oleophilicity and hydrophobicity.

The OHM sponge was embedded in epoxy resin for ultramicrotome to prepare thin (cross-sectional) sections for characterization, especially of the hard-soft interfaces in this composite system.

Backscattered electron (BSE) imaging in SEM shows compositional contrast, which highlights the spatial distribution of the iron oxide coating. It is more difficult to differentiate between the various carbon components (polyurethane, graphite, resin). However, graphite has a distinct Raman spectral signature, which can be used to map its spatial distribution. A combined SEM-Raman correlative approach is necessary to properly map the coating at the microscale.

The coating uniformity and characteristics are observed on the nanoscale by TEM analysis. A typical annular dark field image (ADF) (Figure 1c) shows an interfacial region between the sponge (left), the coating (middle) and the resin used in the sample preparation (right). Presence of iron in the coating results in a brighter contrast. In some places, as shown in (d) large graphite flakes were explicitly visible. To confirm the structure of the graphite and iron oxide, selected area electron diffraction in (e) was obtained from a region in (d). Selected area diffraction ring patterns match well with predictions for iron oxide nanoparticles and graphite.

Accurate spatial distribution of iron oxide $\left(\mathrm{Fe}_{3} \mathrm{O}_{4}\right)$ and graphite is addressed by using simultaneously acquired EDS and EELS maps, as shown in Figure 2. The ADF image (a) shows a typical region of coating along the sponge. The EELS map (b) confirms the presence iron oxide nanoparticles along the strand and embedded in the graphite flake. The degree of $\mathrm{sp}^{2}$ clustering ( $\pi$ bond) is reflected in the low-loss range of 
the spectrum and can be used to distinguish between the various types of carbon bonds in the graphite, resin and sponge. As shown in (c) presence of graphitic, $\mathrm{sp}^{2}$ bonding, is clearly observed in the characteristic graphite flakes, but also along the strand where the iron oxide nanoparticles are. The distribution of graphitic character is evidence of the embedment of the nanoparticles within the graphite, which forms the bulk of the coating. Typical low-loss spectra are shown (d) from two distinct regions, \#1 from the large graphite flake and \#2 from the polyurethane sponge region. We can clearly see a peak at 7 $\mathrm{eV}$ in the spectrum \#1, attributed to the $\pi$ bonding, unique to graphite.

The aforementioned results highlight the chemical and physical properties that contribute to the exceptional performance of OHM sponge in oil recovery. Ongoing Environmental SEM (ESEM) studies are being performed to observe the dynamics of fluid-solid interfaces of both OHM sponge and of flat model systems. These operando experiments will help us to further understand the hydrophobic/oleophilic nature of the sponge. We are also exploring ways to tailor the OHM sponge to target other aqueous pollutants, such as nutrients and heavy metals. This presentation will emphasize the need for proper sample preparation and correlative-multimodal characterization to study the OHM sponge and more broadly other materials with hard-soft interfaces. [6]

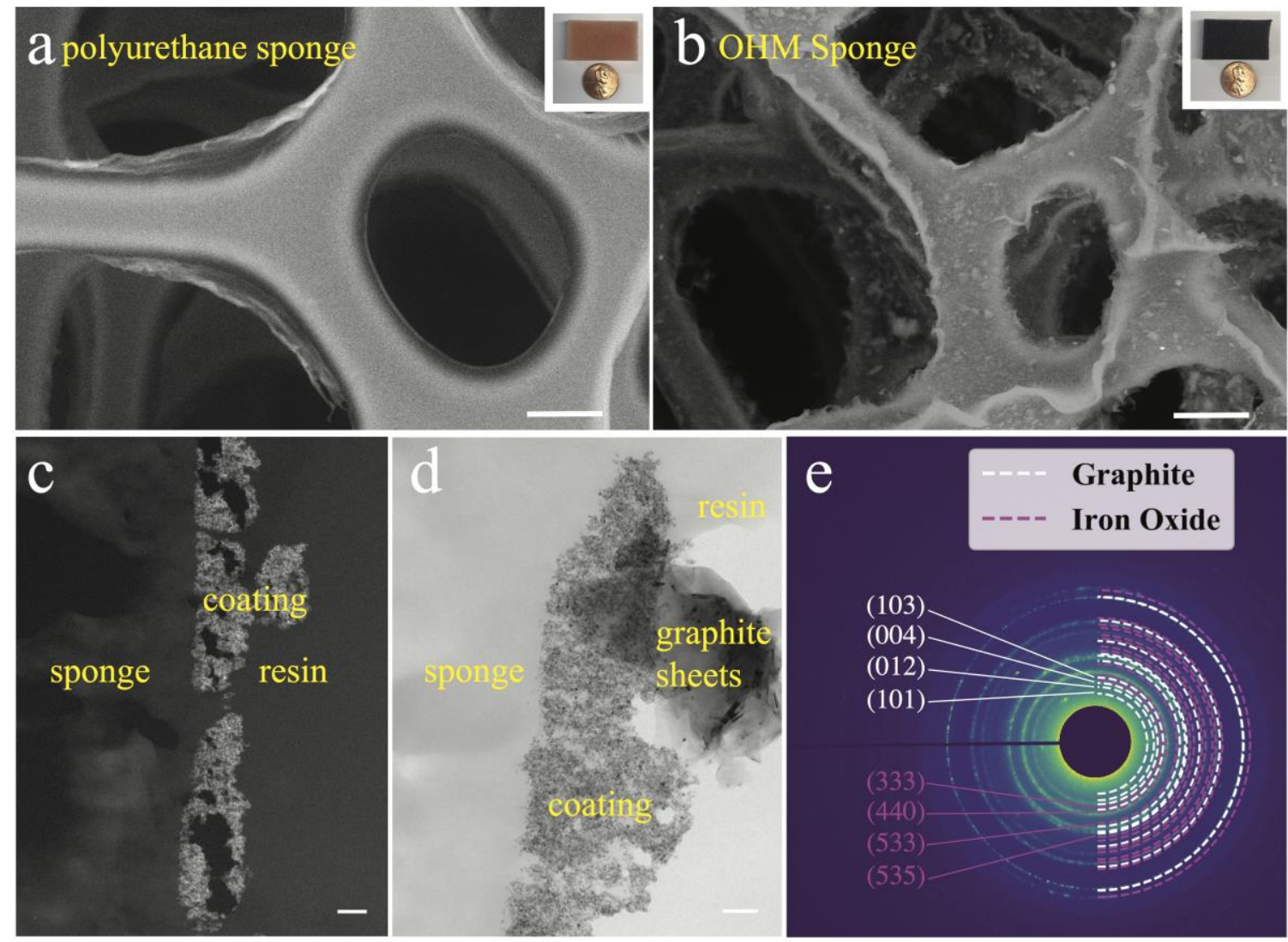

Figure 1. SEM of a sponge before (a) and after (b) coating with nanocomposite. Insets show the visual appearance of the sponge. Interface between sponge, coating and graphite flakes is depicted in the ADF image (c) and BF-TEM (d). Selected Area Diffraction Pattern (e) from (d) confirms the structure of iron oxide and graphite. Scale bar in 1a,b is $50 \mu \mathrm{m}, 1 \mathrm{c}$ is $200 \mathrm{~nm}$, and $1 \mathrm{~d}$ is $100 \mathrm{~nm}$. 

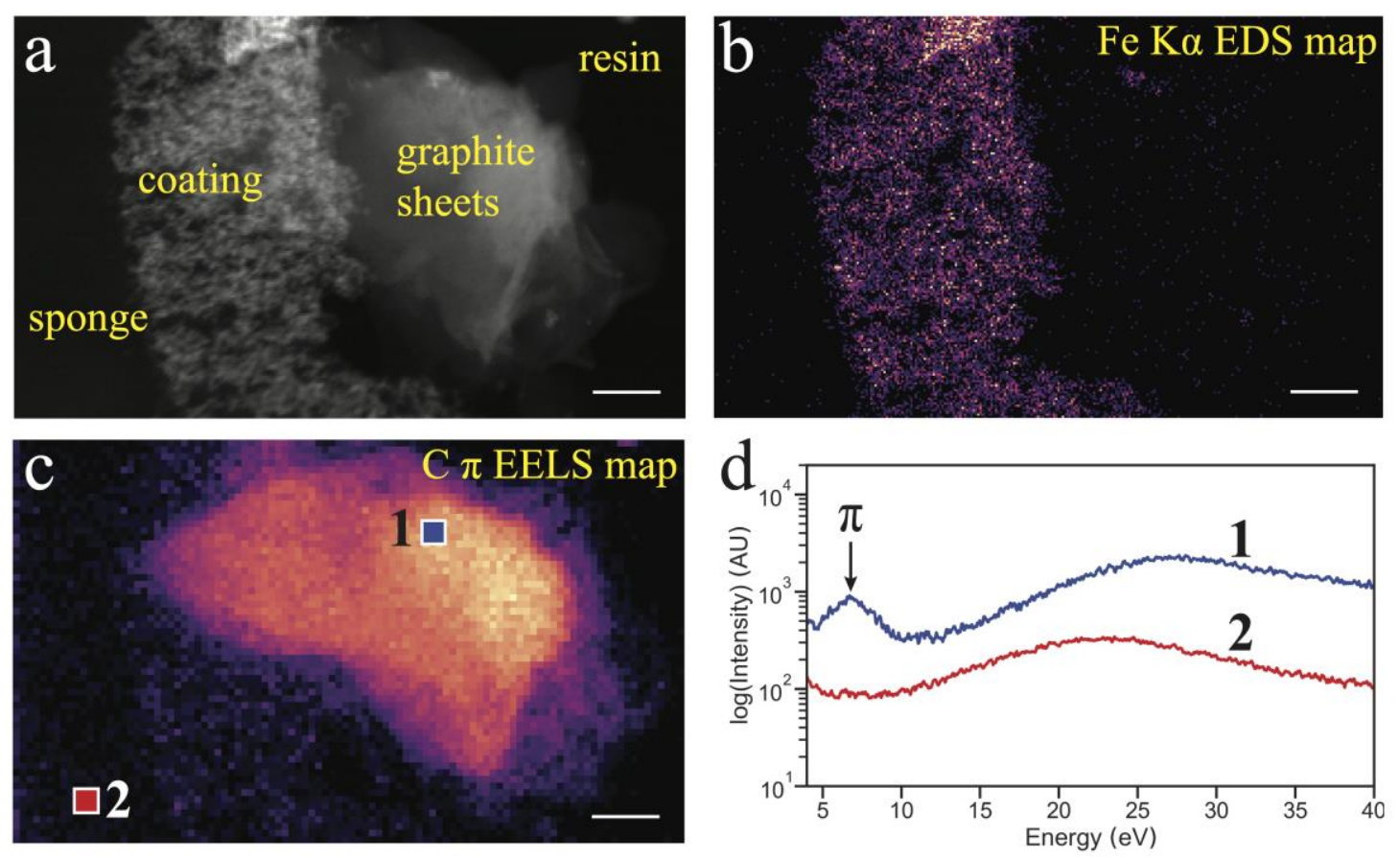

Figure 2. (a) ADF image from a region comprising the sponge, the coating, graphite sheets and the resin. (b) EDS map of Fe distribution within the region in (a). (c) Simultaneously acquired EELS map of the same area qualitatively showing regions that contains graphite. (d) EELS spectrum from points 1 and 2 in (c). The signature peak from $\pi$ bond in graphite used to map out the areas with graphite is indicated in the spectrum 1. Scale bar is $100 \mathrm{~nm}$.

\section{References}

[1] Nandwana, V. et al., Ind. \& Eng. Chem. Res. (2020), 59, 23, 10945-10954.

[2] V. Dravid. US Patent 62/788,347, Filed: Jan 4, 2019.

[3] Lee, Z. et al., Nano Lett. (2009), 9, 9, 3365-3369.

[4] Li, Q. et al., Science (2018), 359, 6376, 669-672.

[5] Rossetti, L. et al., Nature Mater (2017), 16, 664-670.

[6] The research related to oxide nanostructured architecture was supported by the National Science Foundation under Grant No. DMR-1929356. This work made use of the EPIC, SPID, and BioCryo facilities of Northwestern University's NUANCE Center, which has received support from the Soft and Hybrid Nanotechnology Experimental (SHyNE) Resource (NSF ECCS-1542205); the MRSEC program (NSF DMR-1720139) at the Materials Research Center; the International Institute for Nanotechnology (IIN); the Keck Foundation; and the State of Illinois, through the IIN. It also made use of the CryoCluster equipment, which has received support from the MRI program (NSF DMR-1229693). 